\title{
An exploratory clinical trial on the safety and efficacy of iNKT cell and PD-1+CD8+ T cell-based innovative immunotherapy in patients with lung adenocarcinoma
}

\author{
Xiaobo Cheng \\ Shanghai Public Health Clinical Center \\ Jing Wang \\ Shanghai Public Health Clinical Center \\ Chenli Qiu \\ Shanghai Public Health Clinical Center \\ Yanling Jin \\ Shanghai Public Health Clinical Center \\ Bili Xia
}

Shanghai Public Health Clinical Center

Ran Qin

Shanghai Public Health Clinical Center

Huiliang Hu

Shanghai Public Health Clinical Center

Jia Yan

Shanghai Public Health Clinical Center

Xiaoyan Zhang ( $\nabla$ zhangxiaoyan@shphc.org.cn )

Shanghai Public Health Clinical Center https://orcid.org/0000-0002-0023-2771

Jianqing Xu

Shanghai Public Health Clinical Center

\section{Research}

Keywords: iNKT cell, PD-1+CD8+ T cell, immunotherapy, safety, efficacy, patients with lung adenocarcinoma, exploratory clinical trial

Posted Date: September 18th, 2020

DOI: https://doi.org/10.21203/rs.3.rs-70176/v1 
License: (c) (i) This work is licensed under a Creative Commons Attribution 4.0 International License. Read Full License 


\section{Abstract}

Background and purpose

The antitumor activities of invariant natural killer T (iNKT) cells and CD8+ T cells have been confirmed. As tumor-specific CD8+ T cells, PD-1+CD8+ T cells kill tumor cells relatively effectively and specifically. We aimed to assess the feasibility, toxicity, antitumor efficacy, and biological correlates of response in patients with advanced lung adenocarcinoma treated with iNKT cells and PD-1+CD8+ T cells for the first time.

\section{Methods}

We performed a single-arm, prospective, single-center exploratory clinical trial that is ongoing and registered at ClinicalTrials.gov (NCT03093688). We expanded autologous iNKT cells and PD-1+CD8+ T cells in vitro using our patented technology. Patients were infused with autologous iNKT cells $(1 \times 108$ $\sim 1 \times 1010), \mathrm{PD}-1+\mathrm{CD} 8+\mathrm{T}$ cells $(1 \times 107 \sim 1 \times 109)$ and dendritic cells (DCs) $(5 \times 105)$ every $3-5$ weeks, which was considered 1 cycle. The study was conducted from March 1, 2017. The primary endpoint were safety and objective tumor response.

Results

An analysis of the completed first stage is reported here. A total of 3 consecutive patients were enrolled and received autologous iNKT cell and PD- $1+C D 8+T$ cell-based immunotherapy ( $\geq 4$ cycles). The first patient received 16 cycles. Computed tomography (CT) examination revealed a stable disease (SD) response after 4 cycles and a progressive disease (PD) response after 11 cycles. The overall survival (OS) time was 20 months. The second patient received 10 cycles. CT examinaton revealed an SD response after 4 cycles and a PD response after 9 cycles. The OS time was 10 months. The third patient received 6 cycles. CT examination revealed an SD response. The OS time was 5.5 months. The patients suffered from only fever, chills, soreness, headache, dizziness, fatigue, sweating, a sick feeling, nausea, vomiting and shortness of breath and received symptomatic treatment.

\section{Conclusions}

iNKT cell and PD-1+CD8+ T cell-based immunotherapy showed a manageable tolerability profile and effective antitumor activity and was selected as a prospective antitumor therapy.

Trial registration

The protocol had retrospectively registered at clinical trial.gov, Unique identifer: NCT03093688, date of registration: March 01, 2017;

https://www.clinicaltrials.gov/ct2/show/NCT03093688?term=NCT03093688\&rank=1 


\section{Full Text}

This preprint is available for download as a PDF.

\section{Figures}

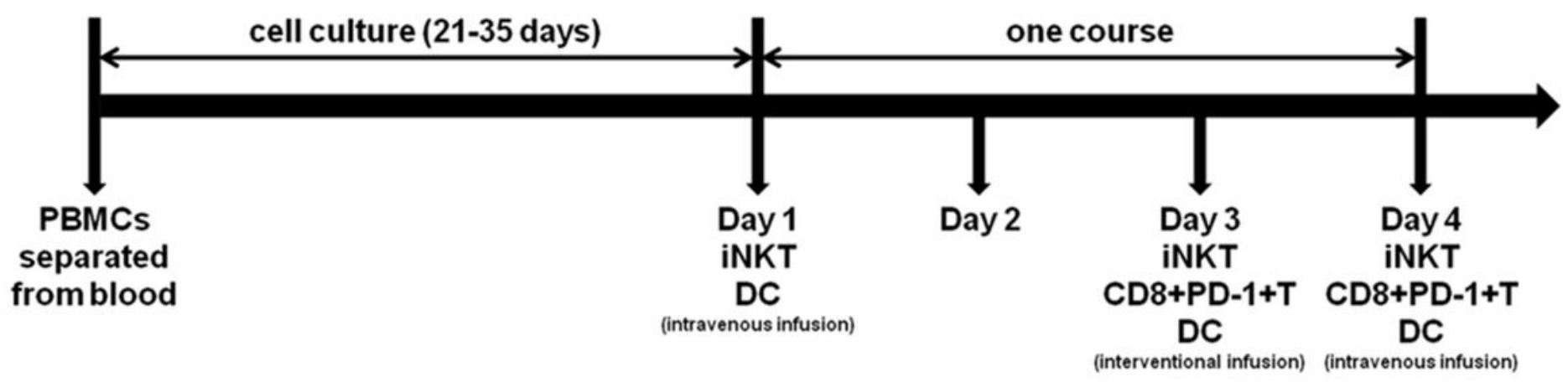

Figure 1

Design of one course of cell transfusion. After all cultured cells were harvested, DCs mixed with iNKT cells were transfused via intravenous infusion on the first day, and DCs mixed with iNKT cells and CD8+PD-1+ T cells were transfused via interventional infusion on the third day and via intravenous infusion on the fourth day. 
A

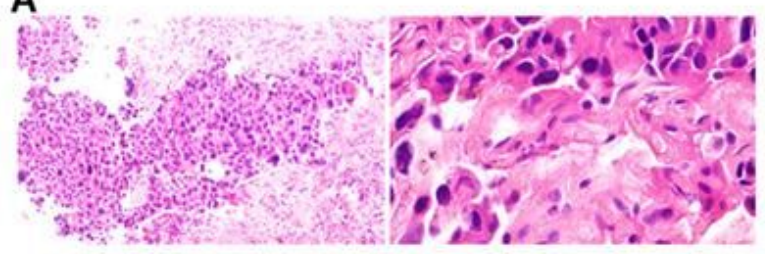

C

Baseline
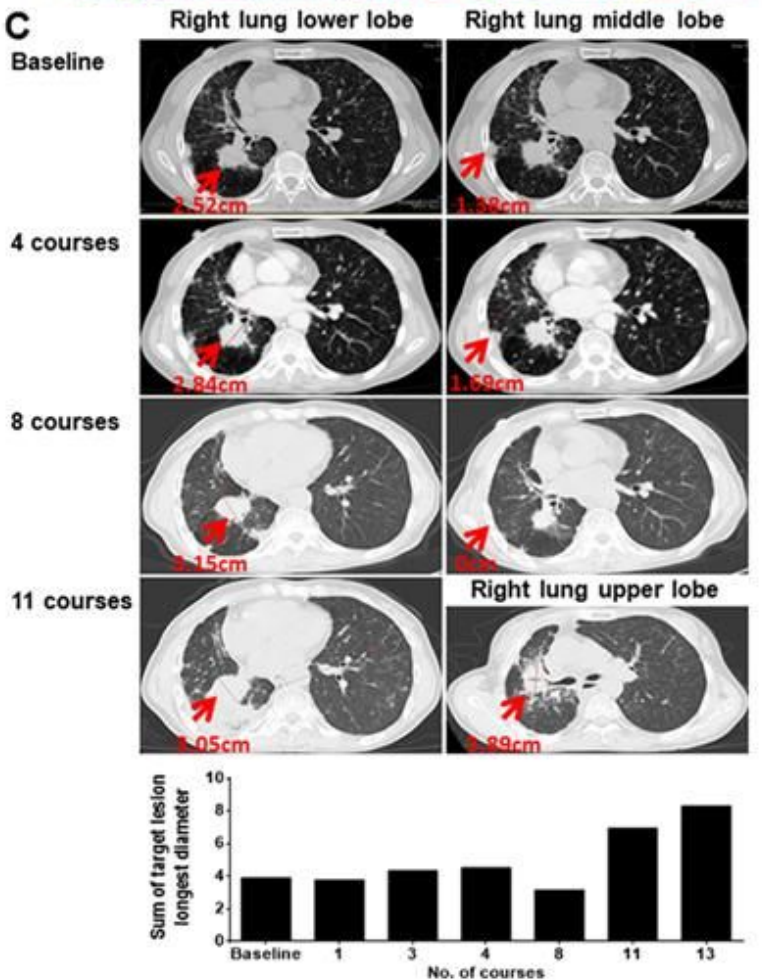

B

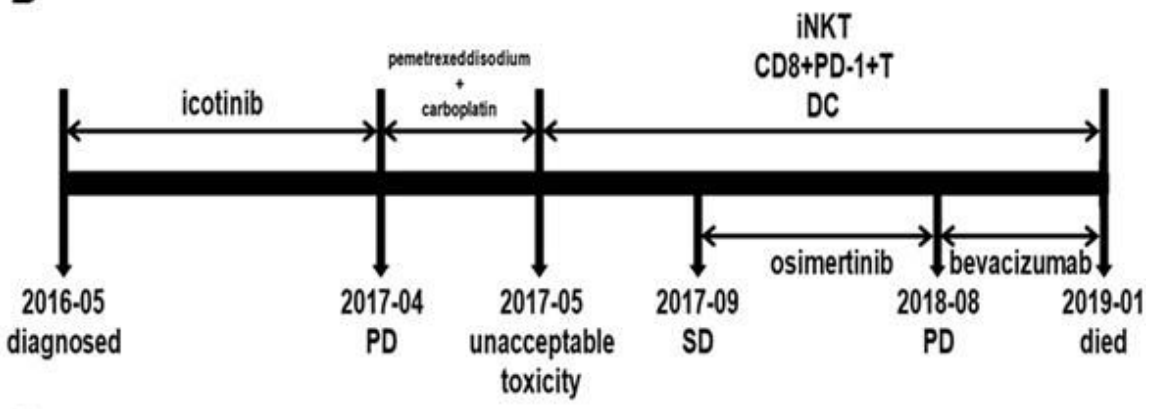

D

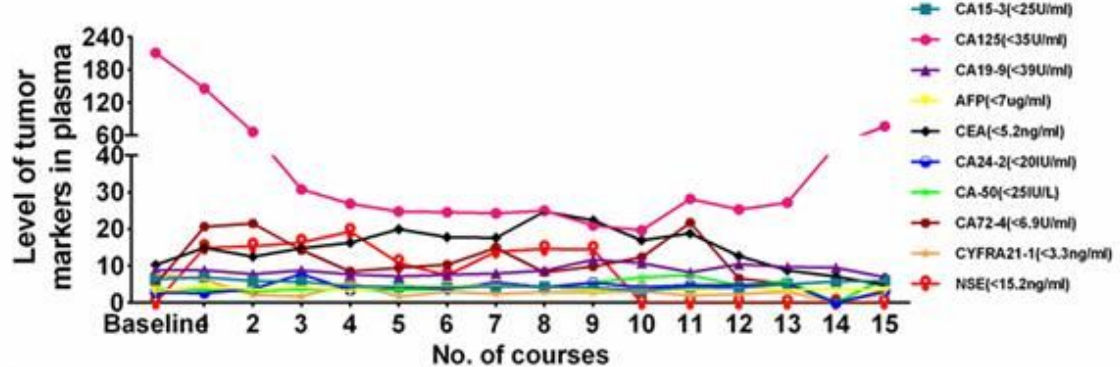

E

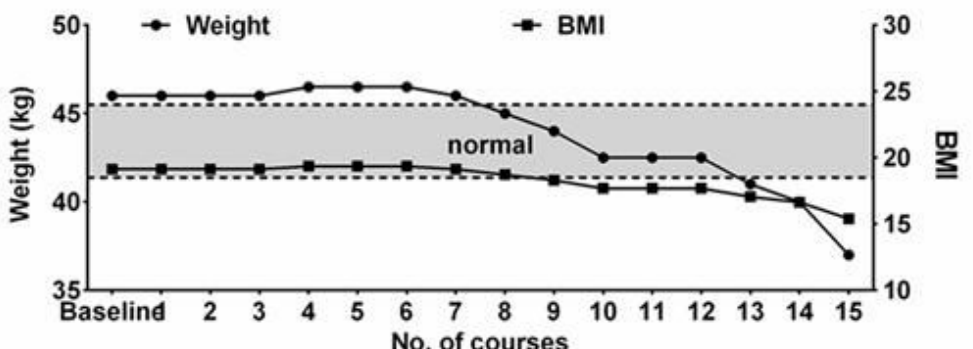

Figure 2

Case 1 Pathology of the patient (A). Treatment of the patient (B). Changes in the tumor burden (C). Changes in tumor markers (D). Changes in weight (E). 
A
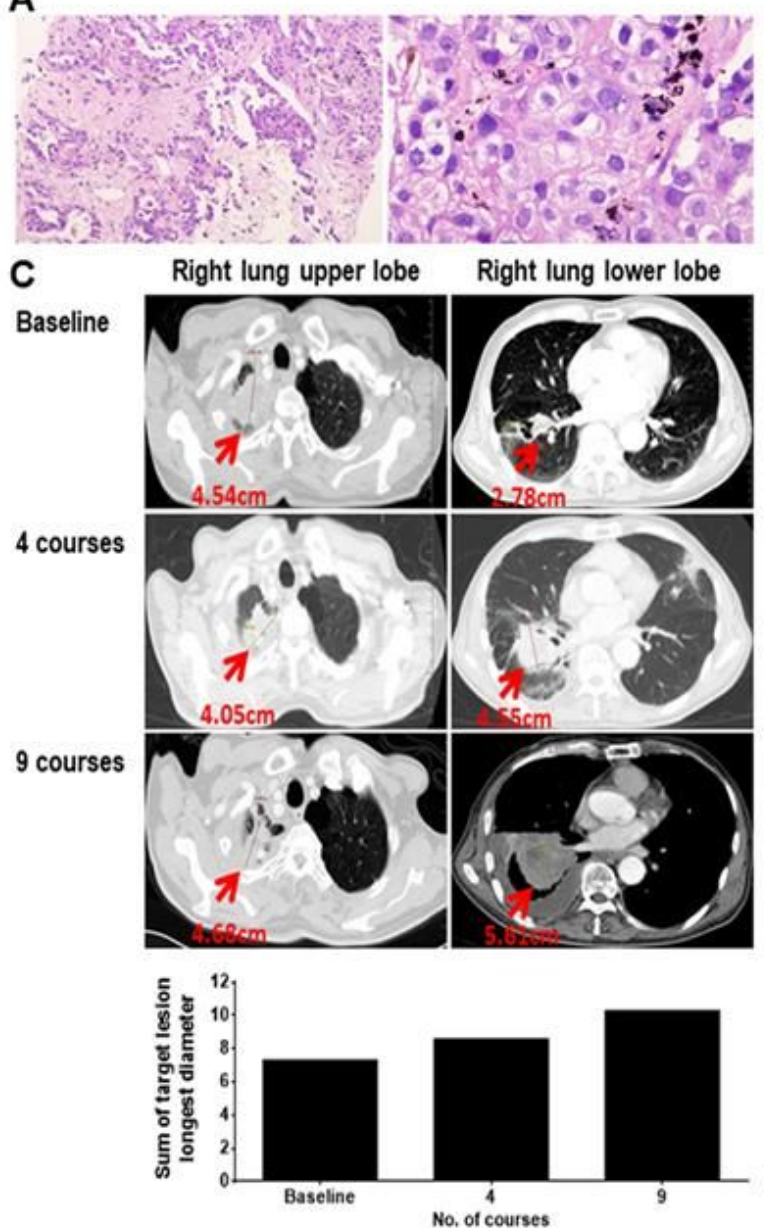

B

INKT

CD8+PD-1+T

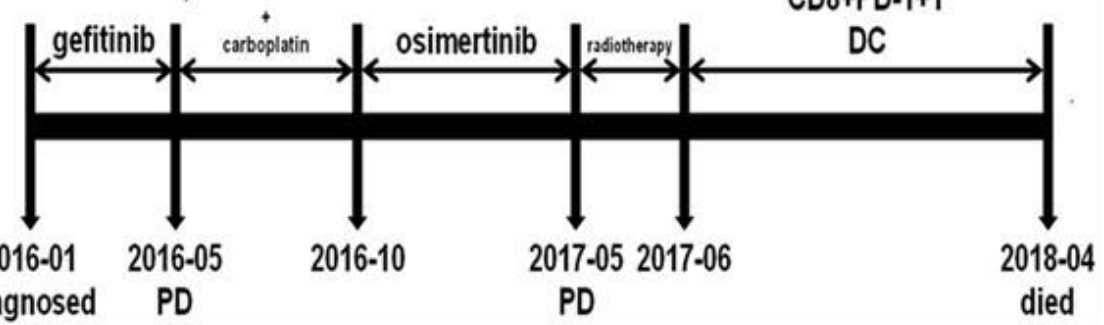

D

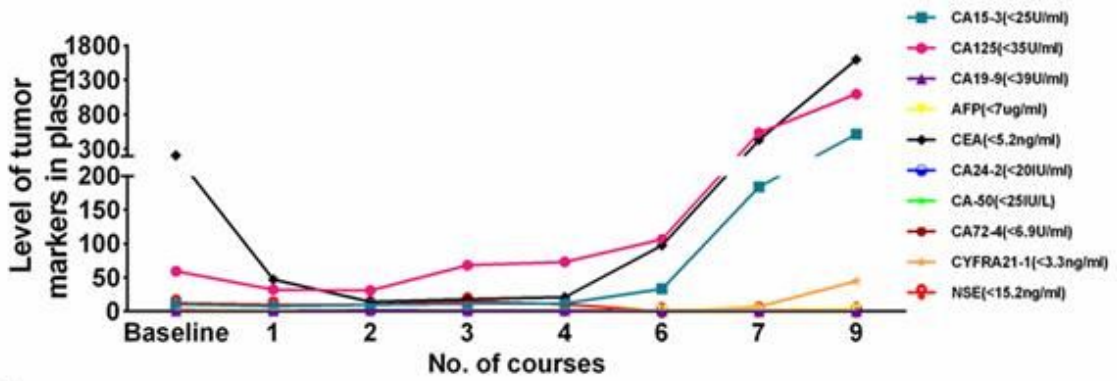

E

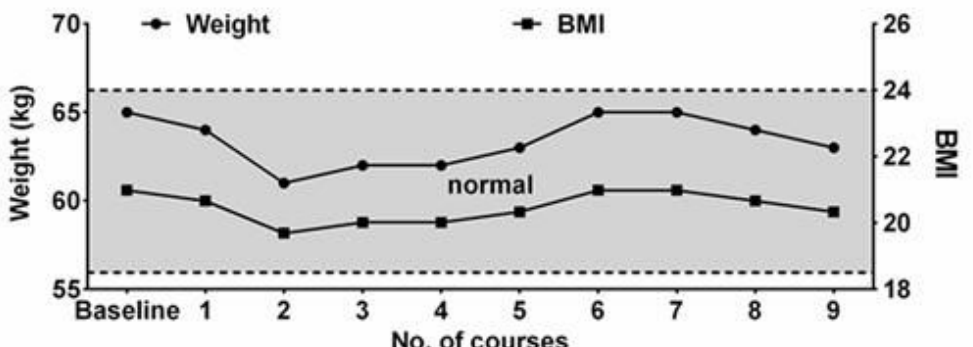

Figure 3

Case 2 Pathology of the patient (A). Treatment of the patient (B). Changes in the tumor burden (C). Changes in tumor markers (D). Changes in weight $(E)$. 
A

C

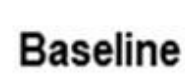

\section{4 courses}


B

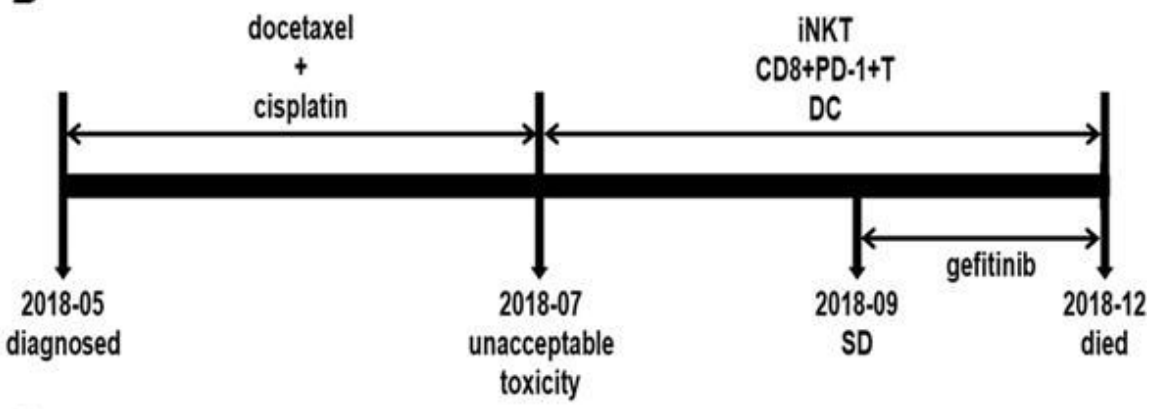

D

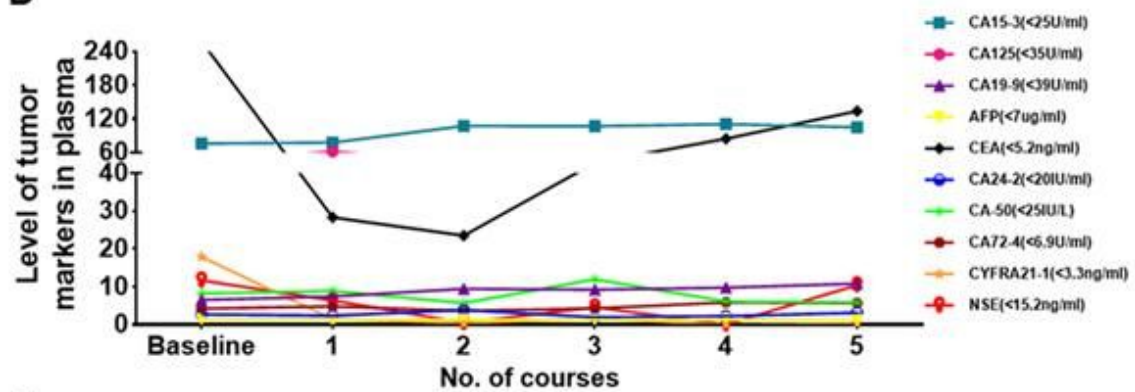

E

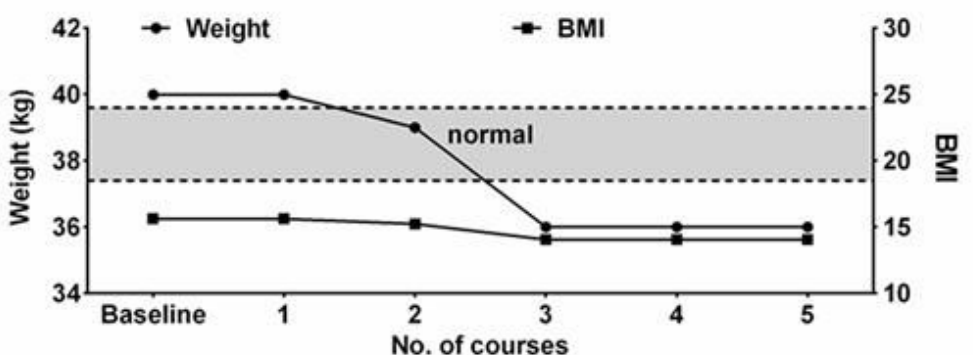

Figure 4

Case 3 Pathology of the patient (A). Treatment of the patient (B). Changes in the tumor burden (C). Changes in tumor markers (D). Changes in weight (E). 